\title{
Antibiotic Resistance to Escherichia coli among Urine Culture-Positive Patients in a Tertiary Care Hospital in Nepal: A Descriptive Cross- sectional Study
}

Anu Kushwaha, ${ }^{1}$ Khilasa Pokharel, $^{2}$ Anuj Raj Kadel ${ }^{3}$

'Department of General Practice and Emergency, Kathmandu Medical College and Teaching Hospital, Sinamangal, Kathmandu, Nepal, ${ }^{2}$ Department of Microbiology, Kathmandu Medical College and Teaching Hospital, Sinamangal, Kathmandu, Nepal, ${ }^{3}$ Department of Medical Education, Kathmandu Medical College and Teaching Hospital, Sinamangal, Kathmandu, Nepal.

\section{ABSTRACT}

Introduction: Urinary tract infection is treated by the antibiotic sensitivity pattern of uropathogens in our population. Present infection cases have been showing an increase in resistance to the current first-line of antibiotics. The objective of this study is to determine the resistance of antibiotics in Escherichia coli in a tertiary care center.

Methods: This is a descriptive cross-sectional study done in Kathmandu Medical College Teaching Hospital from October 2018 to February 2019. The sample size was calculated, and convenient sampling was done. Patients with urine culture positive (i.e., Colony-forming unit >105) were included in the study. All positive samples were tested for antibiotics sensitivity and resistance. The resistance to the antibiotics was recorded using Statistical Package for Social Sciences version 20. Point estimate at $95 \%$ confidence interval was calculated along with frequency and proportion for binary data.

Results: Out of 100 samples, the highest number of organisms isolated was Escherichia coli, 71 (71\%). Escherichia coli showed the highest resistance to drugs like Norfloxacin, 37 (52.11\%), AmoxicillinClavulanic acid $37(52.11 \%)$, followed by Co-trimoxazole 32 (45.1\%), Ceftriaxone, 24 (33.8\%), and Ciprofloxacin $23(32.3 \%)$.

Conclusions: Escherichia coli showed the highest resistance to commonly used antibiotics like Norfloxacin, Amoxicillin-Clavulanic acid, Co-trimoxazole, Ceftriaxone, and Ciprofloxacin.

Keywords: antibiotic; drug resistance; urinary tract infections.

\section{INTRODUCTION}

Urinary tract infection (UTI) is defined as the presence of pathological bacteria in the urinary system from the kidney to the urethra. After respiratory tract infection, urinary tract infection is the most common infection in our society.

UTI in the genito-urinary tract without any instrumentation is called uncomplicated UTI. In the structurally and functionally abnormal urinary tract and associated with instrumentation, it is called complicated UTI. ${ }^{1}$ Severe form of UTI is due to Escherichia coli (E. coli). ${ }^{2}$ Klebsiella, Proteus, Pseudomonas, Staphylococcus, Enterobacter, and Enterococci are other organisms causing UTI. There is an increased risk of developing antimicrobial resistance due to the unregulated use of antibiotics worldwide. ${ }^{3}$ Empirical treatment for UTI in patients has to be formulated time and again, given the increasing tendency of antibiotics resistance in microorganisms.

This study aims to find the prevalence of urinary tract

Correspondence: Dr. Anu Kushwaha, Department of General Practice and Emergency Medicine, Kathmandu Medical College and Teaching Hospital, Sinamangal, Kathmandu, Nepal. Email: anukushwaha@gmail.com, Phone: +977-9841315359. 
Kushwaha et al. Antibiotic Resistance to Escherichia coli among urine culture-positive patients in a Tertiary Care Hospital in Nepal...

infections caused by E. Coli among urine culturepositive patients and identify which antibiotics E. Coli has developed resistance against.

\section{METHODS}

A descriptive cross-sectional study was carried out in the Clinical Microbiology Laboratory of Kathmandu Medical College and Teaching Hospital. Ethical approval was taken from the Institutional Review Committee (IRC), Ref no:2311201812, KMCTH. All urine culture-positive patients were included in the study, and patients with culture-negative are excluded from the study.

An antibiotic sensitivity test of $E$. coli isolated from clinical specimens against different antibiotics was performed on Muller Hinton Agar (MHA) by the KirbyBauer method's standard disk diffusion technique. ${ }^{4}$

Convenient sampling was done, and the sample size was calculated using the formula:

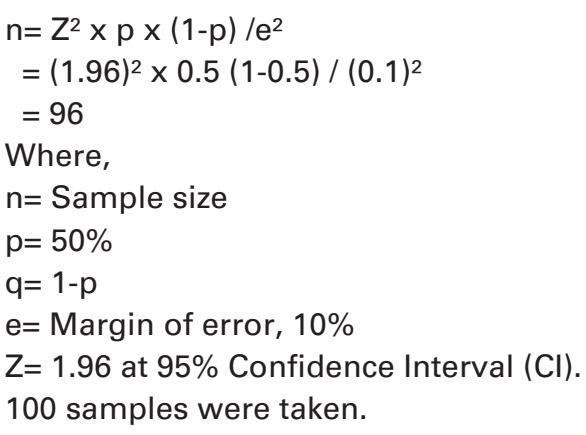

Data analysis was done using Statistical Package for the Social Sciences-20 version.

\section{RESULTS}

Out of the 100 urine culture samples which tested positive for an organism, Escherichia coli was isolated in $71(71 \%)$ of them. E. coli showed resistance to drugs like Norfloxacin, 37 (52.11\%), Amoxicillin-Clavulanic acid, followed by Co-trimoxazole, 32 (45.1\%), Ceftriaxone, 24 (33.8\%) and Ciprofloxacin, 23 (32.3\%) (Table 1).

\begin{tabular}{|ll|}
\hline \multicolumn{2}{|l|}{ Table 1. Antibiotic resistance to E. coli. } \\
\hline Antibiotics name & $\begin{array}{l}\text { Resistance seen in } \\
\text { urine culture samples } \\
\text { in which E. Coli was } \\
\text { isolated } \mathrm{n}(\%)\end{array}$ \\
Amikacin & $9(12.67)$ \\
Ampicillin+Sulbactam & $2(2.82)$ \\
Amoxicillin+Clavulanic acid & $37(52.11)$ \\
Amoxicillin & $0(0)$ \\
Azithromycin & $7(9.86)$ \\
Clavulanic acid & $2(2.82)$ \\
\hline Ceftazidime & $1(1.41)$
\end{tabular}

\begin{tabular}{|ll|} 
Cefsulodine & $29(40.8)$ \\
Cefixime & $1(1.41)$ \\
Ciprofloxacin & $23(32.3)$ \\
Chloramphenicol & $1(1.41)$ \\
Co-trimoxazole & $32(45.1)$ \\
Cloxacilln & $0(0)$ \\
Ceftriaxone & $24(33.8)$ \\
Cefotaxime & $2(2.82)$ \\
Gentamicin & $2(2.82)$ \\
Imipenem & $4(5.63)$ \\
Meropenem & $6(8.45)$ \\
Nitrofurantoin & $0(0)$ \\
Norfloxacin & $37(52.11)$ \\
Polymyxin B & $0(0)$ \\
Piperacillin+Tazobactam & $1(1.41)$ \\
Tobramycin & $3(4.23)$ \\
Total Samples & $71(100)$ \\
\hline
\end{tabular}

Hence highest resistance was present for Norfloxacin and Amoxicillin-Clavulanic acid. No resistance was seen for Amoxicillin, Cloxacillin, Nitrofurantoin, and Polymyxin B.

\section{DISCUSSION}

This study evaluated the antibiotics resistance pattern of $E$. coli. In this study, the maximum number of patients in the age group of 21-30 was UTI. This may be attributed to the fact that 21 to 30 is a sexually active age group. Similar patterns were also observed in studies done by Mohamad Akram et al. in AligarhIndia. ${ }^{3,4,5}$

The higher incidence of UTI among females is likely due to anatomical and hormonal variation among the two sexes, making the female gender more susceptible to the infection. This discrepancy due to gender was consistent with studies done in another center. ${ }^{5,6}$ Unsurprisingly, E. coli was the commonest organism isolated from the urinary samples. ${ }^{7-9}$

E.coli showed high resistance to Amoxyclav, Norfloxacin, Cefsulodin, Ceftriaxone, Ciprofloxacin, and Co-trimoxazole low resistance to Gentamicin, Meropenem, Imipenem, and Amikacin. These results were almost similar to the findings reported by Oge Kale Olunalan Timothy et al. and SiddiqueM et al.,8

Uropathogens are increasingly becoming resistant to several commonly used antibiotics. Thus, resistance and sensitivity patterns of microorganisms causing infectious diseases, including UTI, are necessary to provide a proper guideline. This increased resistance may be a consequence of unregulated antibiotics use and warrants strict rules and regulations on antibiotics' consumption and prescription.

\section{CONCLUSIONS}


Kushwaha et al. Antibiotic Resistance to Escherichia coli among urine culture-positive patients in a Tertiary Care Hospital in Nepal...

E. coli was the most common bacteria isolated from urinary tract infection, followed by K. pneumonia. These uropathogens are increasingly becoming resistant to several commonly used antibiotics, controlled by formulating a proper guideline for treating physicians. This increased resistance may be a consequence of unregulated antibiotics use and warrants strict rules and regulations on antibiotics' consumption and prescription.

\section{Conflict of Interest: None.}

\section{REFERENCES}

1. Alsohaili SA, Alharahshed MH, Almshagbeh MA, Alkhawaldeh RA, Alkhawaldeh WM. Bacterial pathogen in urinary tract infection and antibiotic resistance pattern in Zaraqa-Jordan. European scientificjournal. 2015;11(12):171-7. [Full Text]

2. Gupta P. Study of antibiotic resistance pattern in uropathogens at a tertiary hospital care hospital. J Evol Med Dent Sci. 2012 Oct;1(4):321-7. [Full Text]

3. Pradhan B, Pradhan SB. Prevalence of Urinary Tract Infection and Antibiotic Susceptibility Pattern to Urinary Pathogens in Kathmandu Medical College and Teaching Hospital, Duwakot. Birat Journal of Health Sciences. 2017;2(1):134-7. [Full Text | DOI]

4. Clinical and Laboratory Standard Institute. Performance standards for antimicrobial disk Susceptibility testing: seventeen information supplement [Internet]. Clinical and Laboratory Standard Institute: USA; 2007 Jan [cited 2020 May]. 177p. Available from: https://www.academia. edu/36252064/CLSI. [Full Text]

5. Akram M, Sahid M, Khan AU. Etiology and resistance patterns of community-acquired urinary tract infections in JNMC Hospital Aligrh, India. Ann Clin Microbiol Antimicrob. 2007 March;6:4. [ubMed | Full Text | DOI]

6. Khaleel M, Mustafa M, Kulkarni P. Antibiotic resistance patterns of community-acquired urinary tract infection in Tertiary care teaching hospital, Hyderbad. Indian journal of basic and applied medical research. 2014 Sep;3(4):142-51. [Full Text]

7. Khan N, Khan MS. Prevalence of antimicrobial resistance in bacterial isolates causing urinary tract infection in patients attending at IIMS \& R Hospital, Lucknow. International Journal of Life Sciences Scientific Research. 2016 Jan;2(1):1-8. [Full Text]

8. Cunha MA, Assuncao GLM, Medeiros IM, Reis M. Antibiotic resistance pattern of urinary tract infection in a Northeastern Brazilian Capital. Rev Inst Med Trop Sao Paulo. 2016 Feb;58:2. [uuMed $\mid$ Full Text $\mid$ DOI]

9. Timothy OO, Olusesan FJ, Adosoia BO, Temitayo AA, David $\mathrm{EO}$, Ige OO. Antibiotic resistance pattern of bacterial isolates from urinary tract infection among hospital and out patients at tertiary health facility in South Western Nigeria.Ann Trop Med Public Health. 2014;7(2):130-5. [․ㅏll Text]

This work is licensed under a Creative Commons Attribution 4.0 International License. The images or other third party material in this article are included in the article's Creative Commons license, unless indicated otherwise in the credit line; if the material is not included under the Creative Commons license, users will need to obtain permission from the license holder to reproduce the material. To view a copy of this license, visit http://creativecommons.org/licenses/by/4.0/ 